Original Article

\title{
PARTIAL AMORPHIZATION OF POORLY-SOLUBLE SIMVASTATIN USP USING MEDIA MILLING IN SYNERGISM WITH SPRAY-DRYING
}

\author{
HARITA R. DESAI', ARCHANA B. RAJADHYAX², PURNIMA D. AMIN*
}

1,2Department of Pharmaceutics, Bombay College of Pharmacy, Kalina, Santacruz-East, Mumbai 400098, India, ${ }^{3}$ Department of Pharmaceutical Sciences and Technology, Institute of Chemical Technology, N P Marg, Matunga-East, Mumbai 400019, India Email: purnima.amin@yahoo.co.in

Received: 12 Aug 2020, Revised and Accepted: 21 Sep 2020

\begin{abstract}
Objective: The objective of the current study was to explore top down methods of size reduction like high speed homogenisation and media milling in synergism with spray drying in amorphization and solubility enhancement of BCS Class II antilipidemic drug Simvastatin USP.

Methods: Spray-dried micronized simvastatin USP was formulated by homogenisation and media milling of drug suspension in optimized stabilizer solution. Stabilizer combination, duration of homogenisation and ball milling and drug: stabilizer ratio was optimized. The obtained dispersion was transformed into solid powder using spray drying. The obtained Spray-dried micronized Simvastatin USP was evaluated for visual morphology, Infrared spectroscopy, Differential scanning calorimetry, in vitro drug release studies, X-Ray diffractometry, Scanning electron microscopy, contact angle measurement, solubility studies, dispersibility studies and intrinsic dissolution rate testing.
\end{abstract}

Results: Spray-dried micronized simvastatin USP was found to show amorphization of crystalline Simvastatin USP as confirmed by the absence of drug peak in Differential scanning calorimetry and lowered signal intensity in X-Ray diffraction studies. Spray-dried micronized Simvastatin USP was found to show enhanced drug hydrophilicity and solubility as confirmed by lowering in contact angle and increase in solubility and ease of dispersibility observations. In vitro dissolution testing and intrinsic dissolution rate testing were found to show an increase in drug release from $11 \%$ to $79 \%$ and $4 \mathrm{mg} \mathrm{min}^{-1} \mathrm{~cm}^{-2}$ to $17 \mathrm{mg} \mathrm{min}^{-1} \mathrm{~cm}^{-2}$ for drug and Spray-dried micronized Simvastatin USP respectively.

Conclusion: Media milling in synergism with spray-drying was found to be a prospective solubility enhancement technique for poorly-soluble Simvastatin USP.

Keywords: Simvastatin, Solubility enhancement, Media milling, Spray drying, Stabilizer, Amorphization, Contact angle, Homogenization (C) 2020 The Authors. Published by Innovare Academic Sciences Pvt Ltd. This is an open access article under the CC BY license (http://creativecommons.org/licenses/by/4.0/) DOI: http://dx.doi.org/10.22159/ijpps.2020v12i11.39373. Journal homepage: https://innovareacademics.in/journals/index.php/ijpps.

\section{INTRODUCTION}

Different active pharmaceutical ingredients discovered via high throughput screening or combinatorial chemistry have been attempted in designing of dosage form for oral administration as the oral route is very convenient in terms of patient administration of the dosage form [1, 2]. The poor solubility characteristics of pharmacologically active lipophilic compounds greatly affect their bioavailability and pose a challenge to formulation scientists in designing suitable bioavailable dosage forms [3-5]. This issue is often seen for BCS Class II drugs exhibiting high permeability but poor solubility [6]

The differences observed in the physicochemical attribute of solubility for solid drugs are due to differences in solid state properties mainly the long range periodicity of the molecules [7] Pharmaceutical solids existing in amorphous form are high energy systems which exhibit higher solubility and faster dissolution velocities and hence are preferred over crystalline forms [8] Different arrangements of lattice structure give rise to distinct crystalline forms of drugs exhibiting variable aqueous solubility. Hence modulations in drug crystalline characteristics can pave a way for modulations in solubility of the drug [9].

Use of solvents and surfactants in solubility enhancement can lead to precipitation and toxicity issues resulting in the need to conduct more stringent toxicity studies [10-12]. Further several innovative approaches like cyclodextrin complexation, solid dispersions, dendrimeric conjugation, liquisolid compaction, self microemulsifying drug delivery systems etc attempted for solubility enhancement have exhibited lesser commercial viability owing to the high cost, complex formulation steps and analytical procedures $[13,14]$.

Particle size reduction of poorly soluble drugs has been widely explored to enhance drug solubility and thus dissolution rate $[15,16]$
Size reduction to lower size ranges and nanonization to size less than $500 \mathrm{~nm}$ is accompanied by increase in surface area which consequently aids to enhance the drug saturation solubility and hence the dissolution rate as per the Nernst Brunner/Noyes Whitney equation and the Ostwald-Freundlich equation $[17,18]$. For solubility enhancement, techniques like High shear homogenisation (HSH), Media milling, Jet milling (JM) etc. can be explored for micronization and nanonization of pharmaceutical drugs [19-21].

Simvastatin USP; a BCS Class II drug is used widely as a cholesterol and lipid lowering agent in hypercholesteremia and dyslipidemia [22]. Structure of Simvastatin is shown in fig. 1. Simvastatin USP exhibits its antilipidemic activity by generating a $\beta$-hydroxy acid form as a metabolite that inhibits the rate limiting step of conversion of HMG Co-A to mevalonate in cholesterol synthesis. Simvastatin USP shows poor aqueous solubility and hence low bioavailability of less than $5 \%$ which makes designing of bioavailable dosage forms a challenging task [23].

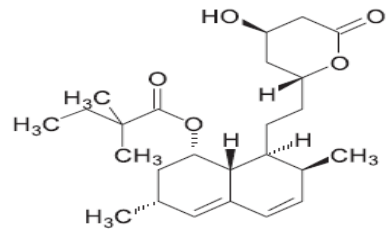

Fig. 1: Structure of simvastatin USP drug

The primary focus of the current research work was to evaluate the effect of top down methods of size reduction like high speed 
homogenization and media milling in synergism with spray-drying on the crystalline characteristics, solubility and dissolution rate of Simvastatin USP.

\section{MATERIALS AND METHODS}

Simvastatin USP was a gift from Biocon Ltd, Bangalore, India Lutrol F68, Lutrol F127 and Vitamin E TPGS were gifted by BASF India, Turbhe, Mumbai. HPMC E15 was gifted by Coloron India, Mumbai. Sodium lauryl sulphate was purchased from SD Fine Chemicals Ltd, Mumbai, India. Mannitol was gifted by DFE Pharma, Mumbai, India. A laboratory-scale media mill of volume $500 \mathrm{ml}$ fabricated by S. F. Engineering works, India was used for size reduction of drug. Remi Motors RQ-127 homogenizer was used for homogenization. JISL LSD 48 Spray drier was used for spray drying.

\section{Formulation of Simvastatin micronized suspension}

The stabilizer system for micronized Simvastatin USP suspension was optimized by determining the contact angle of surfactant solutions like Lutrol F68, Lutrol F127, HPMC E15, PVPK30, Sodium lauryl sulphate and Kolliphor ®) TPGS (d-alpha tocopheryl polyethylene glycol 1000 succinate) with drug in a concentration of $0.5 \%$ and $0.25 \%$ as single surfactant system and in combination with Sodium lauryl sulphate respectively using Kruss contact angle meter and sessile drop method [25]. Coarse suspension of Simvastatin USP in distilled water was prepared using the obtained optimised stabiliser system in the ratio of 5:1 and 2:1. The formula for coarse suspension subjected to homogenization and ball milling is as shown in table 1. Balls of two different dimensions; 22 balls of $19 \mathrm{~mm}$ and 27 balls of $14 \mathrm{~mm}$ were considered for the ball milling process conducted occupying more than $50 \%$ of ball mill volume.

Table 1: Formula for coarse suspension to be homogenized and ball milled

\begin{tabular}{|c|c|c|c|c|}
\hline & $\begin{array}{l}\text { Quantity in } \% \mathrm{w} / 100 \mathrm{ml} \\
\text { for ratio 5:1 }\end{array}$ & $\begin{array}{l}\text { Quantity for } 300 \mathrm{ml} \text { (in } \\
\text { gms) for ratio } 5: 1\end{array}$ & $\begin{array}{l}\text { Quantity in } \% \mathrm{w} / 100 \\
\text { ml for ratio 2:1 }\end{array}$ & $\begin{array}{l}\text { Quantity for } 300 \mathrm{ml} \text { (in } \\
\text { gms) for ratio } 2: 1\end{array}$ \\
\hline Simvastatin USP & 2.5 & 7.5 & 2.5 & 7.5 \\
\hline Lutrol F68 & 0.25 & 0.75 & 0.625 & 1.875 \\
\hline Sodium Lauryl Sulphate & 0.25 & 0.75 & 0.625 & 1.875 \\
\hline Distilled water q. s & q. $\mathrm{s}$ to $100 \mathrm{ml}$ & q. $\mathrm{s}$ to $300 \mathrm{ml}$ & q. $\mathrm{s}$ to $100 \mathrm{ml}$ & q. $\mathrm{s}$ to $300 \mathrm{ml}$ \\
\hline
\end{tabular}

The coarse suspension of Simvastatin USP containing optimised stabiliser system in the ratio of 5:1 was subjected to $4 \mathrm{~h}$ of High speed homogenisation at $800 \mathrm{rpm}$ and $48 \mathrm{~h}$ of ball milling at $21 \mathrm{rpm}$ Particle size of micronized suspension was determined using Malvern Hydro 2000 particle size analyser after every hour Duration of high speed homogenisation and media milling for effective size reduction was optimized using $d(0.9)$ and $D[4,3]$ values of particle size measurement. Based on size observations with 5:1 ratio of drug: stabilizer, the coarse suspension containing Simvastatin USP and the optimised stabiliser system in the ratio of 2:1 was subjected to 1hour of High speed homogenisation at 800 rpm and $24 \mathrm{~h}$ of ball milling at $21 \mathrm{rpm}$. The obtained micronized Simvastatin USP suspension was transformed into solid powder using Spray drying technique and Mannitol as the carrier in drug: carrier ratio of 1:2. Spray drying parameters was optimized to get best yield of amorphized micronized spray-dried drug

\section{Evaluation studies}

\section{Morphology studies}

Morphological details of obtained spray-dried micronized Simvastatin USP were studied and compared to Simvastatin USP drug using Canon power shot 480 digital camera.

\section{FTIR studies}

Characteristic chemical bands for Simvastatin USP drug and spraydried micronized simvastatin USP were studied using Shimadzu miracle IR affinity-1 FTIR spectrophotometer. The spray-dried micronized Simvastatin USP sample was scanned between $400 \mathrm{~cm}^{-1}$ to $4000 \mathrm{~cm}^{-1}$ using air as the standard using attenuated total reflection probe technique.

\section{Differential scanning calorimetry studies}

The melting characteristics of the spray-dried micronized simvastatin USP were studied using Pyris 6 Perkin Elmer Differential scanning calorimeter (DSC). 3-4 mg of the micronized Simvastatin USP was placed in an aluminium pan. The pan was covered with lid and crimped using DSC crimper. The crimped pans were heated against blank crimped pans from $30{ }^{\circ} \mathrm{C}$ to $300{ }^{\circ} \mathrm{C}$ at $10{ }^{\circ} \mathrm{C} / \mathrm{min}$ under nitrogen flow of $17 \mathrm{ml} / \mathrm{min}$ and obtained endothermic peaks were studied.

\section{X-ray diffraction studies}

The crystalline characteristics of spray-dried micronized simvastatin USP were studied using Bruker D8 Advance XRD analyzer. $1.5 \mathrm{gm}$ of spray-dried micronized Simvastatin USP sample was placed on a sample holder and the sample holder was placed on a rotating sample stage. The sample in the holder was placed in the horizontal position and the X-ray tube and detector were moved over the sample simultaneously to cover the 2 -theta angular range from 2 to 50. The crystalline characteristics were studied from the obtained signal plot of intensity versus 2 -theta values.

\section{Contact angle measurement studies}

The wetting tendency of the spray-dried micronized simvastatin USP was determined using Kruss contact angle goniometer by the sessile drop method. Spray-dried micronized simvastatin USP were converted into flat compacts using Hydraulic press and the angle made by a water drop placed horizontally on the flat surface was measured using inbuilt cross wires and optical eyepiece assembly. The wetting tendency of spray-dried micronized Simvastatin USP was compared with Simvastatin USP [24].

\section{In vitro drug release testing}

The in vitro drug release for Simvastatin USP and spray-dried micronized simvastatin USP was studied using USP Dissolution Apparatus II-Paddle type. $40 \mathrm{mg}$ Simvastatin USP and an amount of spray-dried micronized simvastatin USP equivalent to $40 \mathrm{mg}$ (140 $\mathrm{mg}$ ) were weighed and placed in hard gelatin capsules size 00 . The capsules containing spray-dried micronized simvastatin USP were clamped using sinkers and placed in $900 \mathrm{ml}$ of $0.01 \mathrm{M}$ Phosphate buffer pH 7 containing $0.5 \%$ SLS. The dissolution procedure was conducted at $50 \mathrm{rpm}$ at $37 \pm 0.5^{\circ} \mathrm{C}$. Aliquots of $5 \mathrm{ml}$ were taken at 15 , $30,45,60,90$ and $120 \mathrm{~min}$ and filtered using a 0.45 micron filter. 5 $\mathrm{ml}$ of the aliquot sample was replaced with buffer medium to maintain sink conditions. Absorbance of the aliquot samples was measured at $238 \mathrm{~nm}$ using buffer medium as reference. The drug release was calculated from the standard plot of Simvastatin USP in $0.01 \mathrm{M}$ Phosphate buffer pH 7 containing $0.5 \%$ SLS.

\section{Solubility studies}

Solubility studies of Simvastatin USP and spray-dried micronized simvastatin USP were conducted using a constant temperature water shaker bath. $10 \mathrm{mg}$ of Simvastatin USP and spray-dried micronized simvastatin USP equivalent to $10 \mathrm{mg}$ of Simvastatin USP were added to $10 \mathrm{ml}$ water. The dispersion was shaken for $48 \mathrm{~h}$ at $37^{\circ} \mathrm{C}$. The resulting solution was filtered using $0.45 \mu$ filter and the absorbance of the filtrate was determined using ultraviolet spectroscopy at $238 \mathrm{~nm}$. The solubility was calculated from the determined absorbance using standard plot.

\section{Scanning electron microscopy (SEM) studies}

The fine morphological details of the spray-dried micronized simvastatin USP were studied using a Philips XL 30 Scanning 
electron microscope. The samples were loaded on aluminium stub with conductive double sided adhesive tape, coated with gold in an argon atmosphere $(50 \mathrm{~Pa})$ at $50 \mathrm{~mA}$ for 50 secs. The samples were scanned at a voltage of $20 \mathrm{kv}$.

\section{Dispersibility studies}

The aqueous dispersibility of spray-dried micronized simvastatin USP was determined which was then compared to Simvastatin USP. 1g of Simvastatin USP and spray-dried micronized simvastatin USP was added to $100 \mathrm{ml}$ water and the speed and ease of dispersion was observed and compared.

\section{Intrinsic dissolution rate determination}

Intrinsic dissolution of spray-dried micronized simvastatin USP was determined by rotating disc method (Wood's apparatus).
Simvastatin USP sample and spray-dried micronized simvastatin USP were pressed in between the die and punch of the intrinsic dissolution rate paddle assembly to arrive at a constant flat surface area. The paddles were exposed to $0.01 \mathrm{M}$ Phosphate buffer $\mathrm{pH} 7$ containing $0.5 \%$ SLS and the dissolution rate per square centimetre was calculated [25]

\section{RESULTS}

Formulation of Spray-dried micronized Simvastatin USP suspension:

A combination of $0.25 \%$ Lutrol $\mathrm{F} 68$ and $0.25 \%$ sodium lauryl sulphate was found to show maximum wetting as indicated by highest lowering of the contact angle and hence was considered for further micronization studies. The contact angles of single and combination stabilizer solutions are as shown in fig. 2 .

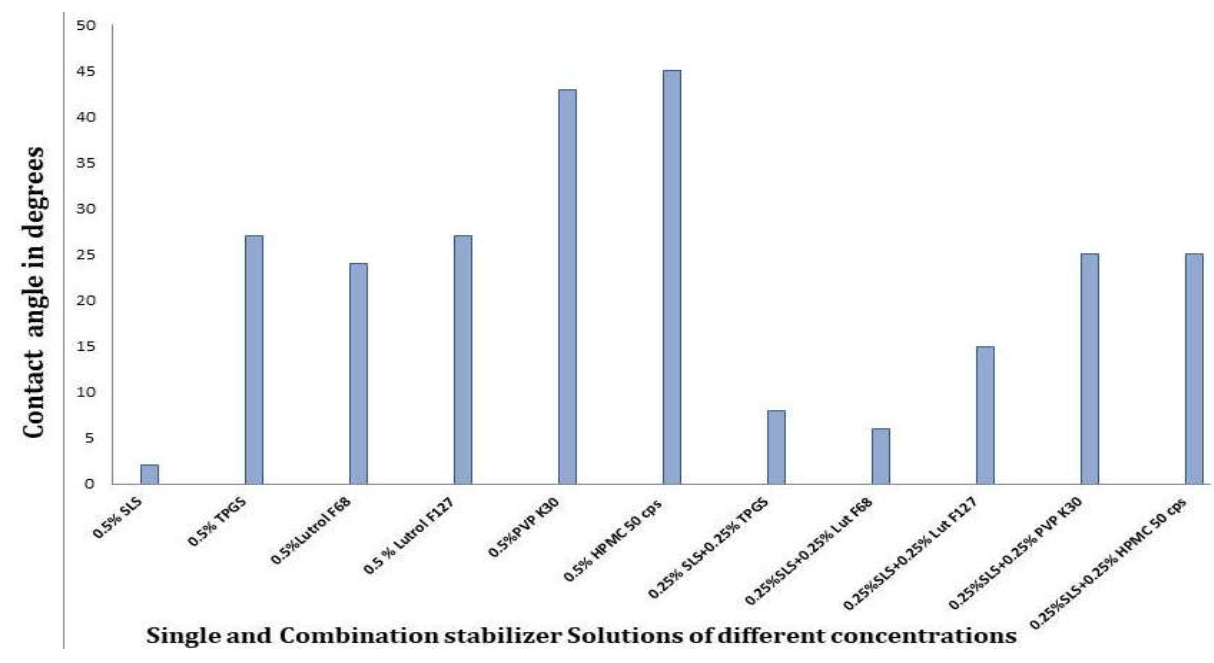

Fig. 2: Contact angle measurement results for stabilizer optimization

For the drug: stabilizer ratio of 5:1, 1 hour of high speed homogenization was found to show the maximum lowering in drug particle size. Homogenization of the suspension for 2,3 and $4 \mathrm{~h}$ was found to show an insignificant effect on the drug particle size. Further ball milling for $24 \mathrm{~h}$ was found to show lowering in particle size whereas $48 \mathrm{~h}$ of milling was found to enhance the size. The particle size of suspension subjected to homogenisation and ball milling for different durations is as shown in table 2. The enhancement in size may be contributed to the initiation of aggregation phenomena owing to soft nature of Simvastatin USP and the relatively lesser amount of stabilizer. Hence 1 hour of high speed homogenisation and 24 hour of ball milling was considered as the optimized duration. Based on the particle size lowering, a drug: stabilizer ratio of 5:1 was found to be effective and considered for further studies.

Table 2: Particle size measurement of suspension after homogenisation and ball milling for different durations

\begin{tabular}{|c|c|c|c|c|}
\hline $\begin{array}{l}\text { Duration of homogenization(HSH)/ } \\
\text { ball milling(BM) }\end{array}$ & $\mathrm{d}(0.1)$ in microns & $\mathrm{d}(0.5)$ in microns & $\mathrm{d}(0.9)$ in microns & $\mathrm{D}[4,3]$ \\
\hline \multicolumn{5}{|l|}{ Drug: stabilizer 5:1 } \\
\hline Plain Simvastatin USP & $11.7 \pm 0.12$ & $27.3 \pm 0.07$ & $73.1 \pm 0.15$ & $41.1 \pm 0.33$ \\
\hline $1 \mathrm{hr} \mathrm{HSH}$ & $6.4 \pm 0.25$ & $15.7 \pm 0.13$ & $34.7 \pm 0.26$ & $19.5 \pm 0.16$ \\
\hline $2 \mathrm{hr} \mathrm{HSH}$ & $4.7 \pm 0.42$ & $14.8 \pm 0.37$ & $36.2 \pm 0.17$ & $18.6 \pm 0.23$ \\
\hline $3 \mathrm{hr} \mathrm{HSH}$ & $4.2 \pm 0.36$ & $14.6 \pm 0.29$ & $36.5 \pm 0.42$ & $18.6 \pm 0.34$ \\
\hline $4 \mathrm{hr} \mathrm{HSH}$ & $4.1 \pm 0.47$ & $14.5 \pm 0.06$ & $36.5 \pm 0.38$ & $18.6 \pm 0.29$ \\
\hline $24 \mathrm{BM}$ & $1.8 \pm 0.16$ & $10.9 \pm 0.23$ & $31.6 \pm 0.09$ & $16.4 \pm 0.43$ \\
\hline $48 \mathrm{~h} \mathrm{BM}$ & $1.9 \pm 0.32$ & $11.9 \pm 0.36$ & $115.4 \pm 0.18$ & $33.9 \pm 0.05$ \\
\hline \multicolumn{5}{|l|}{ Drug: stabilizer 2:1 } \\
\hline $1 \mathrm{hr} \mathrm{HSH}$ & $5.4 \pm 0.34$ & $15.1 \pm 0.18$ & $37.6 \pm 0.21$ & $19.2 \pm 0.35$ \\
\hline $24 \mathrm{hr} \mathrm{BM}$ & $2.8 \pm 0.16$ & $11.9 \pm 0.13$ & $34.5 \pm 0.32$ & $16.2 \pm 0.26$ \\
\hline
\end{tabular}

Data is given in mean \pm standard deviation (SD) for $n=3$

Spray drying of the micronized drug suspension loaded on a carrier at the optimized spray-drying parameters was found to yield free flowing white coloured powder. The optimized spray drying parameters are as shown in table 3 . 
Table 3: Optimized spray drying parameters

\begin{tabular}{ll}
\hline Parameter & Observation \\
\hline Inlet Temperature $\left({ }^{\circ} \mathrm{C}\right)$ & $131.9 \pm 0.2$ \\
Outlet Temperature $\left({ }^{\circ} \mathrm{C}\right)$ & $45 \pm 0.57$ \\
Aspirator & $52 \pm 0.90$ \\
Feed rate (rpm) & $2 \pm 0.12$ \\
Atomizing Pressure (bars) & $2.9 \pm 0.23$ \\
\hline
\end{tabular}

Data is given in mean \pm standard deviation (SD) for $n=3$

Evaluation studies

\section{Morphology studies}

Simvastatin USP drug and spray-dried micronized simvastatin USP was found to show distinct difference in appearance.
Simvastatin USP was observed as shiny white fluffy powder whereas Spray-dried micronized Simvastatin USP was seen as a coarse white powder. Morphological details of Simvastatin USP drug and spray-dried micronized simvastatin USP are as shown in fig. 3.

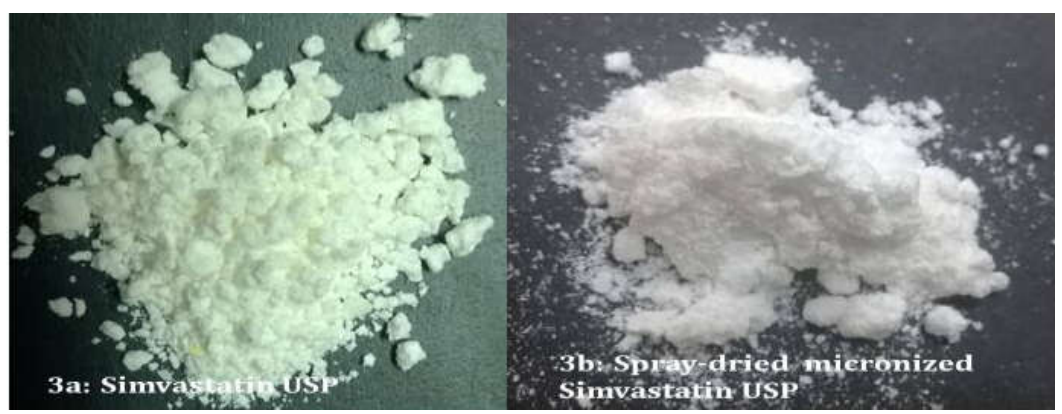

Fig. 3: Visual morphology a. simvastatin USP b. spray dried micronized simvastatin USP

\section{FTIR studies}

FTIR spectra of Simvastatin USP, mannitol and spray-dried micronized simvastatin USP are as shown in fig. 4. FTIR spectra of spray-dried micronized simvastatin USP was found to show bands characteristic for Simvastatin USP and carrier Mannitol indicating no change in the chemical attributes of Simvastatin USP.

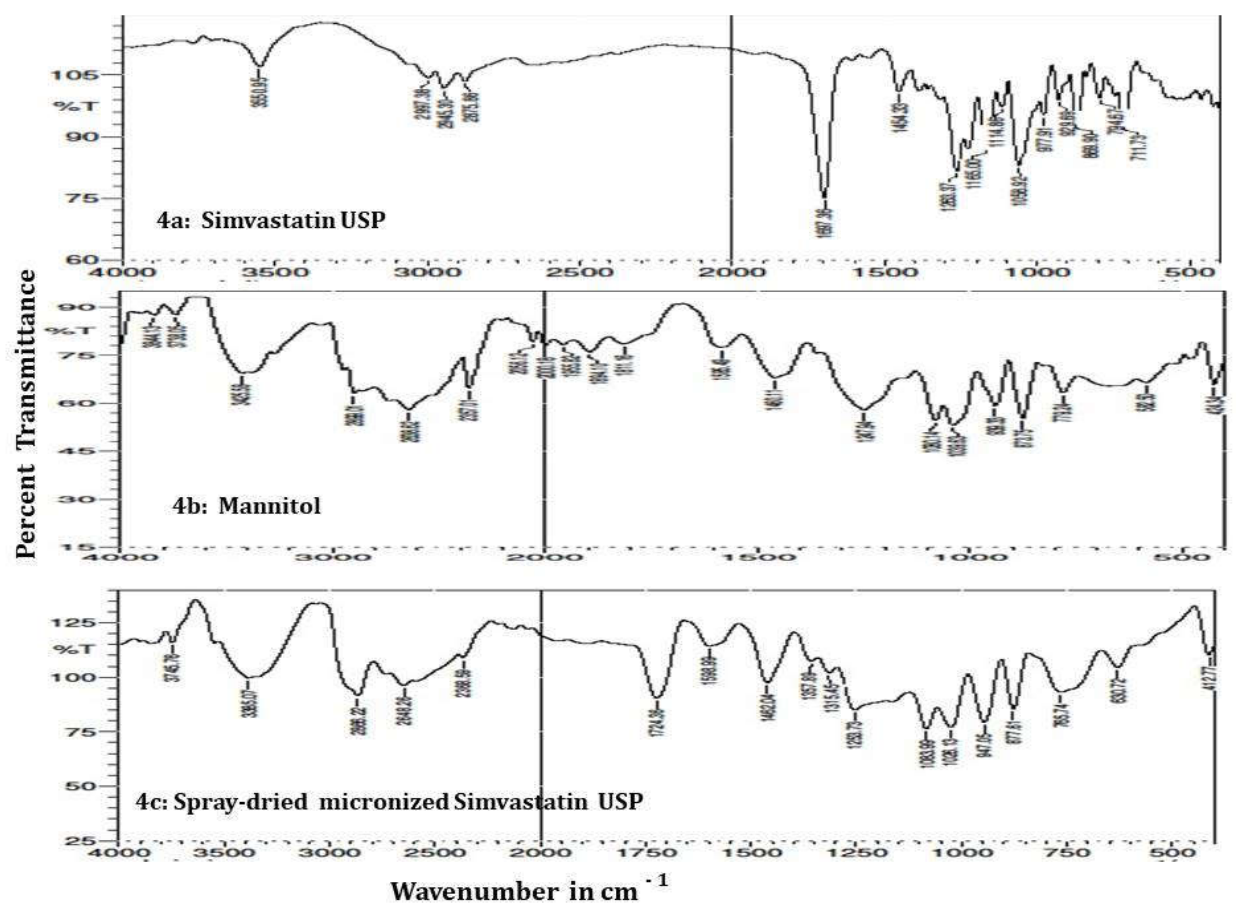

Fig. 4: FTIR Spectra a. simvastatin USP b. mannitol c. spray-dried micronized simvastatin USP 


\section{Differential scanning calorimetry studies}

DSC endotherms for Simvastatin USP, mannitol and spray-dried micronized simvastatin USP are as shown in fig. 5. Simvastatin USP was found to show an endothermic peak at $143.47{ }^{\circ} \mathrm{C}$ whereas
Mannitol was found to show an endothermic peak at $173.24{ }^{\circ} \mathrm{C}$. DSC endotherm of spray-dried micronized simvastatin USP was found to show a single endothermic peak at $168.4^{\circ} \mathrm{C}$ closer to that of carrier Mannitol. No peak for Simvastatin USP was observed thus indicating amorphization of crystalline drug.
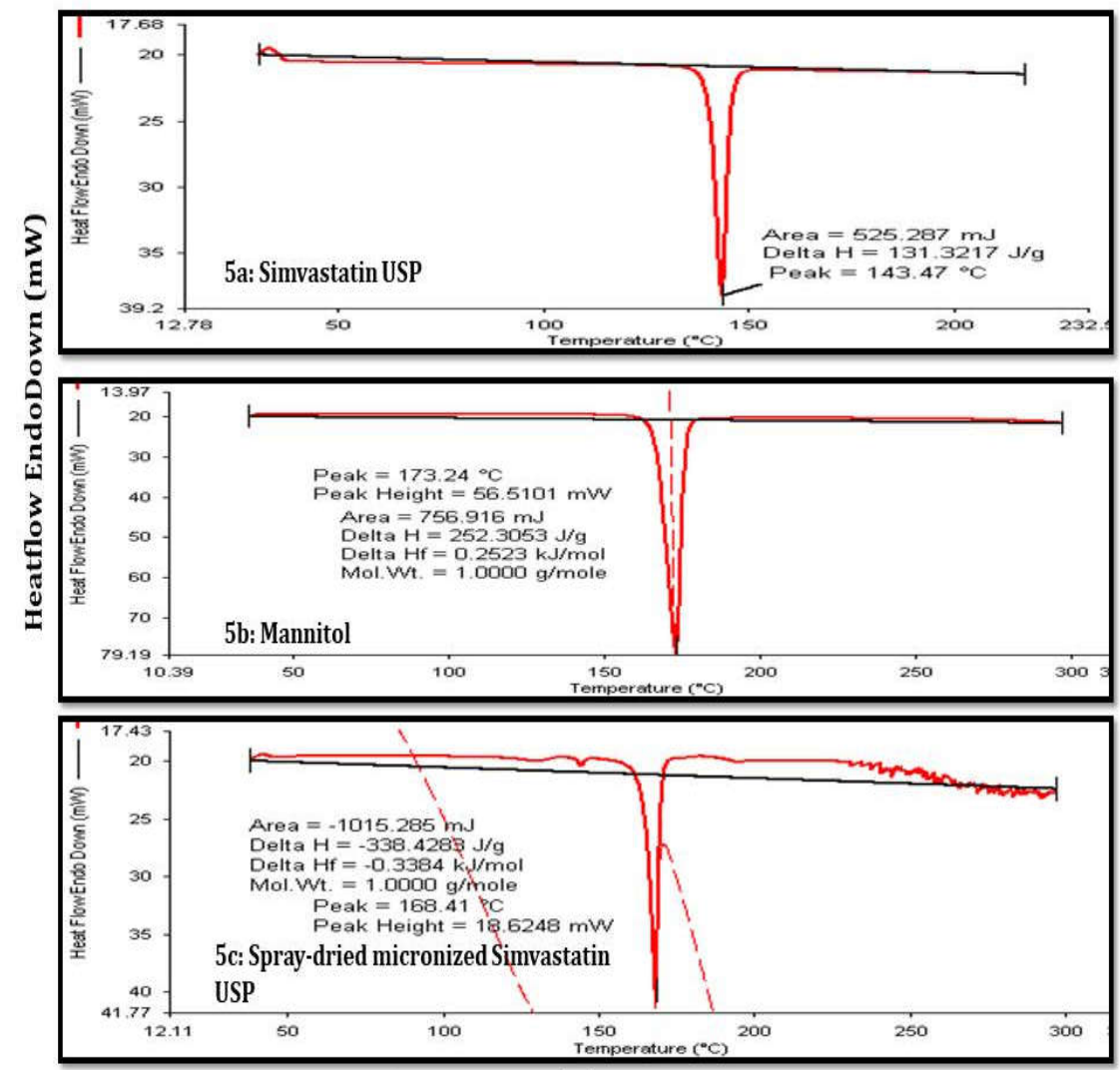

Temperature $\left({ }^{\circ} \mathrm{C}\right)$

Fig.5: DSC endotherm a. simvastatin USP b. mannitol c. spray-dried micronized simvastatin USP
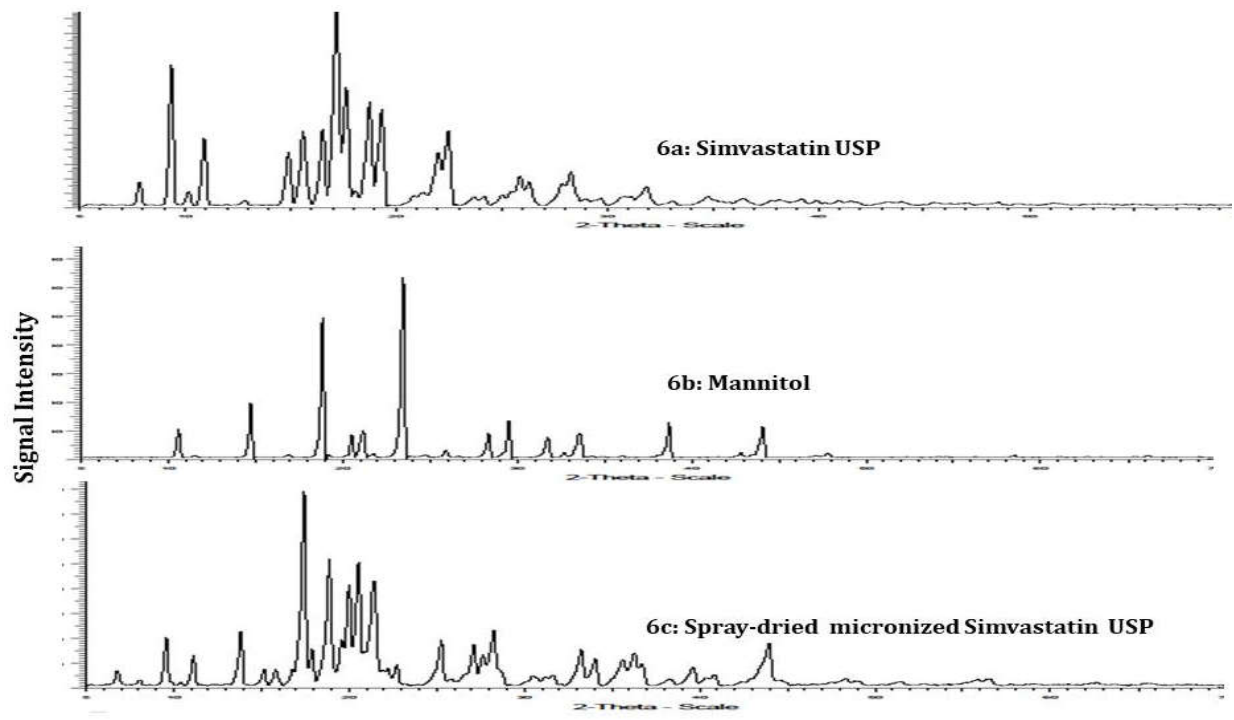

2-Theta Values

Fig. 6: X-ray diffractogram a. simvastatin USP b. mannitol c. spray-dried micronized simvastatin USP 


\section{X-ray diffraction studies}

X-ray diffractograms of Simvastatin USP, mannitol and spray-dried micronized simvastatin USP are as shown in fig. 6. X-Ray diffractogram of spray-dried micronized simvastatin USP was found to show signals at 2 theta values characteristic of Simvastatin USP and Mannitol. A distinct decline in intensity of signals at 2 theta values characteristic of Simvastatin USP was observed in X-Ray diffractogram of spray-dried micronized simvastatin USP thus confirming the amorphization of Simvastatin USP.

\section{Contact angle measurement studies}

Spray-dried micronized Simvastatin USP were found to show drastic lowering of the contact angle from $56^{\circ} \pm 0.23$ for Simvastatin USP to $2^{\circ} \pm 0.10$ thus indicating enhanced hydrophilicity and wetting tendency of spray-dried micronized Simvastatin USP.

\section{In vitro drug release testing}

Spray-dried micronized Simvastatin USP was found to show an increase in drug release of $79 \%$ as compared to plain drug release as observed to be $11 \%$. The in vitro drug release profile for
Simvastatin USP and spray-dried micronized simvastatin USP are as shown in fig. 7.

\section{Solubility studies}

Spray-dried micronized simvastatin USP was found to show increased solubility in water compared to Simvastatin USP owing to enhanced hydrophilicity and increased surface area of the drug. The measured solubility values for Simvastatin USP and spray-dried micronized simvastatin USP are as shown in table 4.

\section{Scanning electron microscopy studies}

The Spray-dried micronized simvastatin USP was found to show porous spherical morphology whereas Simvastatin USP and Mannitol were found to show needle shaped morphology. SEM images for Simvastatin USP, Mannitol and Spray dried micronized simvastatin USP are as shown in fig. 8.

\section{Dispersibility studies}

Spray-dried micronized simvastatin USP was found to disperse completely in water in less than 30 seconds forming homogenous white coloured solution compared to that of plain drug which formed non-wettable heap on the surface of water [fig. 9]

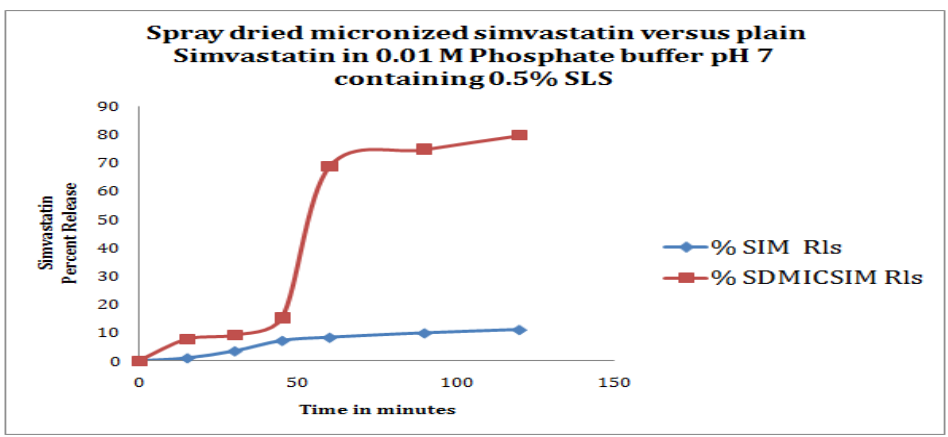

Fig. 7: In vitro drug release of simvastatin USP and spray-dried micronized simvastatin USP (n=3, mean \pm standard deviation)

Table 4: Solubility of simvastatin USP and spray-dried micronized simvastatin USP

\begin{tabular}{ll}
\hline & Solubility in microgram/ml \\
\hline Simvastatin USP & $3 \pm 0.5$ \\
Spray-dried micronized Simvastatin & $500 \pm 0.34$ \\
\hline
\end{tabular}

Data is given in mean \pm standard deviation (SD) for $n=3$
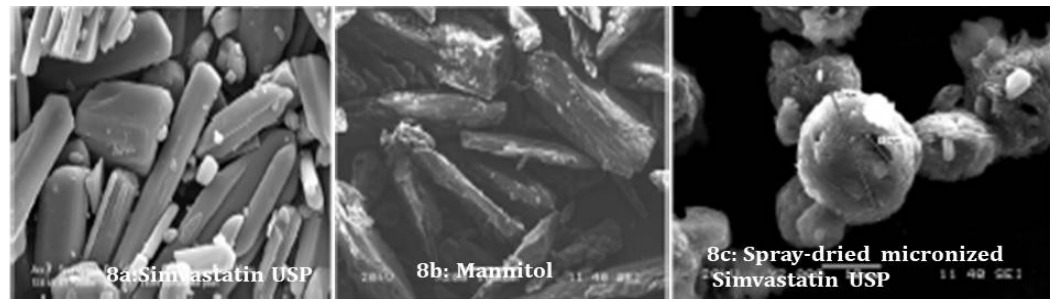

Fig. 8: SEM image a. simvastatin USP b. mannitol c. spray dried micronized simvastatin USP

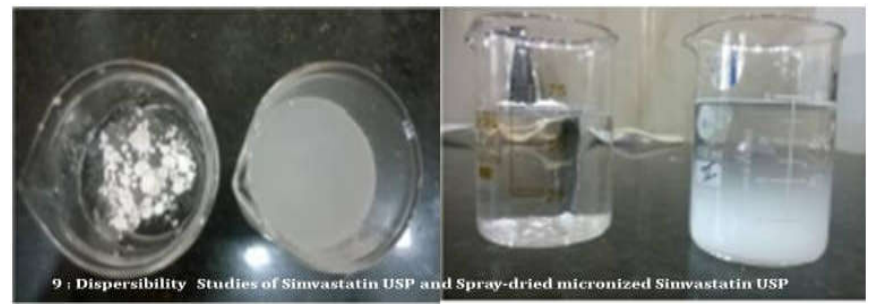

Fig. 9: Dispersibility studies of simvastatin USP and spray-dried micronized simvastatin USP 


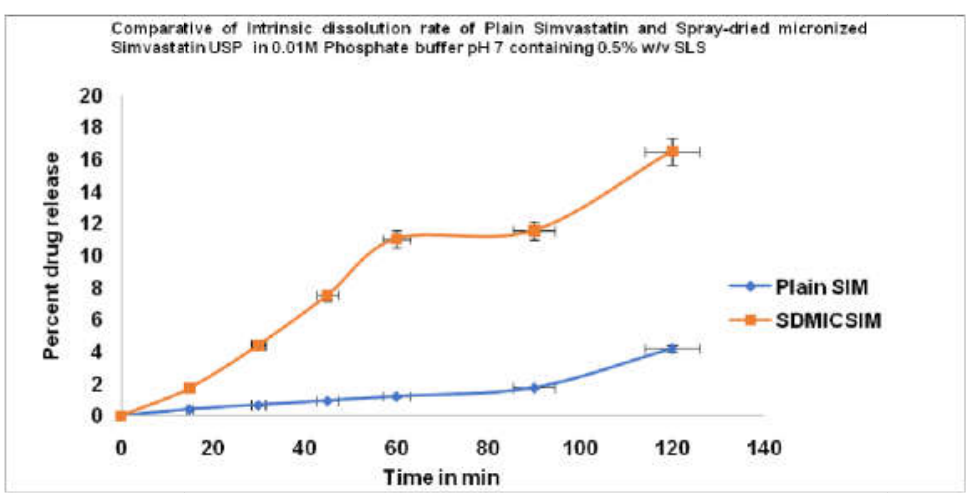

Fig. 10: Intrinsic dissolution rate of simvastatin USP and spray-dried micronized simvastatin USP (n=3, mean \pm standard deviation)

\section{Intrinsic dissolution rate determination}

Spray-dried micronized simvastatin USP was found to exhibit an increased intrinsic dissolution rate of $17 \mathrm{mg} \mathrm{min}^{-1} \mathrm{~cm}^{-2}$ as compared to Simvastatin USP which showed a rate of $4 \mathrm{mg} \mathrm{min}^{-1} \mathrm{~cm}^{-2}$ [fig. 10]

\section{DISCUSSION}

The effect of particle size reduction technique on the drug and selection of a suitable stabilizer system depends on the physical attributes of the drug mainly the hardness of the drug. Combination of non-ionic and ionic surfactant system of Lutrol F68 and sodium lauryl sulphate which was found to exhibit highest lowering in contact angle for Simvastatin USP; aid in stabilization of micronized active Simvastatin USP in the dispersion by steric and electrostatic stabilization [26]. The surfactant combination also aids to influence the solubility by enhanced wetting. The presence of infrared bands characteristic for both Simvastatin USP and Mannitol in Spray-dried micronized simvastatin USP indicates no changes in the chemical properties of the drug. The effect of homogenization and ball milling on the drug crystallinity can be studied by DSC studies. DSC endotherm of Spray-dried micronized simvastatin USP was found to show absence of drug melting peak whereas a single peak was observed at $168.4{ }^{\circ} \mathrm{C}$; a temperature point closer to melting temperature of carrier Mannitol. Thus DSC studies aids to confirm the amorphization effect of high speed homogenisation and ball milling on the crystalline Simvastatin USP. The amorphization was further confirmed by the XRD studies of Spray-dried micronized simvastatin USP which exhibited a distinct decline in intensity of signals at two theta values characteristic for Simvastatin USP. The profound increase in solubility from $3 \pm 0.5$ to $500 \pm 0.34 \mu \mathrm{g} / \mathrm{ml}$ and the increase in drug release from $11 \%$ to $79 \%$ can be attributed to micronization, enhanced wetting and spray-drying treatment of Simvastatin USP. The optimized stabilizer system aids to enhance the hydrophilicity of drug. The micronization process further aids to enhance the surface area available for dissolution in the media. Spray-drying of the micronized drug produces spherical micronized Simvastatin USP drug with high porosity thus aiding the solubilisation effect. Increase in solubilization can be further augmented by the presence of water soluble Mannitol as carrier of the micronized Simvastatin USP [27].

The intrinsic dissolution rate determination mainly aids to determine the specific surface area of the drug solid in contact with medium and available for dissolution. The increase in the Intrinsic dissolution rate of $17 \mathrm{mg} \mathrm{min-1} \mathrm{cm}^{-2}$ for Spray-dried micronized simvastatin USP as compared to $4 \mathrm{mg} \mathrm{min}^{-1} \mathrm{~cm}^{-2}$ for Simvastatin USP aid to confirm the increase in surface area due to drug micronization. The increased surface area increases the saturation solubility of the drug which is further supported by decreased crystal lattice energy and enhanced wetting by surfactant association and the porosity imparted by spray drying process [28].

\section{CONCLUSION}

The solubility of drugs can be improved by manoeuvring the physicochemical attributes. A synergism of particle size reduction and spray drying has been widely studied to enhance solubility. Based on the studies conducted in present research work, it was observed that homogenization and ball milling of poorly-soluble Simvastatin USP in presence of suitable stabilizers followed by spray-drying in presence of carrier Mannitol effectively aided to reduce the size and enhance the hydrophilicity and wetting properties of the drug. Furthermore the treatment was found to amorphize the drug thus enhancing its solubility properties. Thus media milling and spray drying can be attempted as prospective techniques for solubility enhancement for poorly-soluble drugs with versatile therapeutic indications.

\section{ACKNOWLEDGEMENT}

The authors are thankful to Biocon Ltd for providing gift sample of Simvastatin USP drug.

\section{FUNDING}

Nil

\section{AUTHORS CONTRIBUTIONS}

The research theme was finalized under the guidance of Prof Purnima Amin. The research work, data compilation, interpretation and calculations were done by Harita Desai. The manuscript was written and edited by Harita Desai and Archana Rajadhyax.

\section{CONFLICTS OF INTERESTS}

The authors declare no conflicts of interest

\section{REFERENCES}

1. Homayun B, Lin $X$, Choi $H$. Challenges and recent progress in oral drug delivery systems for biopharmaceuticals Pharmaceutics 2019;11:1-29.

2. Basavaraj S, Betageri GV. Can formulation and drug delivery reduce attrition during drug discovery and developmentreview of feasibility, benefits and challenges. Acta Pharm Sin B 2014;4:3-17.

3. Di Costanzo A, Angelico R. Formulation strategies for enhancing the bioavailability of silymarin. Molecules 2019;24:2155.

4. Narayan R, Pednekar A, Bhuyan D, Gowda C, Koteshwara KB Nayak U. A top down technique to improve the solubility and bioavailability of aceclofenac: in vitro and in vivo studies. Int J Nanomed 2017;12:4921-35.

5. Rein MJ, Renouf M, Cruz Hernandez C, Actis Goretta L, Thakkar SK, Pinto M. Bioavailability of bioactive food compounds: a challenging journey to bioefficacy. $\mathrm{Br} \mathrm{J}$ Clin Pharmacol 2013;75:588-602.

6. Tsume Y, Mudie DM, Langutt P, Amidon GE, Amidon GL. The biopharmaceutical classification system: Subclasses for in vivo predictive dissolution (IPD) methodology and IVIVC. Eur J Pharm Sci 2014;57:152-63.

7. Sathisaran I, Dalvi S. Engineering cocrystals of poorly-water soluble drugs to enhance dissolution in aqueous medium. Pharmaceutics 2018;10:108. 
8. Hancock BC, Zografi G. Characteristics and significance of the amorphous state in pharmaceutical systems. J Pharm Sci 1997;86:1-12.

9. Brittain HG. Methods for the characterization of polymorphs and solvates. In: Brittain HG. Editor. Polymorphism in Pharmaceutical Solids. New York: Marcel Dekker; 1999. p. 227-78.

10. Mottu F, Laurent A, Rufenacht DA, Doelker E. Organic solvents for pharmaceutical parenterals and embolic liquids: a review of toxicity data. PDA J Pharm Sci Technol 2000;54:456-69.

11. Bhadoriya S, Madoriya N, Shukla K, Parihar MS. Biosurfactants a new pharmaceutical additive for solubility enhancement and pharmaceutical development. Biochem Pharmacol 2013;2:1-5.

12. Savjani K, Gajjar A, Savjani J. Drug solubility: importance and enhancement techniques. International Scholarly Research Network (ISRN) Pharmaceutics; 2012. p. 1-10.

13. Liversidge GG, Cundy KC. Particle size reduction for improvement of oral bioavailability of hydrophobic drugs. I. Absolute oral bioavailability of nanocrystalline danazol in beagle dogs. Int J Pharm 1995;125:91-7.

14. Jinno J, Kamada N, Miyake M, Yamada K, Mukai T, Odomi M, et al. Effect of particle size reduction on dissolution and ora absorption of a poorly water-soluble drug, cilostazol, in beagle dogs. J Controlled Release 2006;111:56-64.

15. Kesisoglou F, Panmai S, Wu Y. Nanosizing-oral formulation development and biopharmaceutical evaluation. Adv Drug Delivery Rev 2007;59:631-44

16. Srivastava V, Mishra G. Dissolution enhancement of a poorly soluble model drugs using different formulation approaches for immediate release solid dosage form. Asian J Pharm Clin Res 2013;7:63-6.

17. Vengala P, Vanamala R. Nanocrystal technology as a tool for improving dissolution of poorly soluble drug, lornoxicam. Int J Appl Pharm 2018;10:162-8.

18. Van Eerdenbrugh B, Van den Mooter G, Augustijns P. Top-down production of drug nanocrystals: nanosuspension stabilization, miniaturization and transformation into solid products. Int J Pharm 2008;364:64-75.
19. $\mathrm{Hu} \mathrm{J}$, Johnston KP, Williams RO. Nanoparticle engineering processes for enhancing the dissolution rates of poorly water soluble drugs. Drug Dev Ind Pharm 2004;30:233-45.

20. Salazar J, Muller RH, Moschwitzer JP. Combinative particle size reduction technologies for the production of drug nanocrystals. J Pharm 2014;1-14. https://doi.org/10.1155/2014/265754

21. Swain RP, Pendela S, Panda S. Formulation and evaluation of gastro-bilayer floating tablets of simvastatin as immediate release layer and atenolol as sustained release layer. Indian J Pharm Sci 2016;78:458-68.

22. Parmar S, Mishra R, Shirolkar SV. Spherical agglomeration a novel approach for solubility and dissolution enhancement of simvastatin. Asian J Pharm Clin Res 2016;9:65-72.

23. Gupta D, Mandowara V, Patel S, Shelat P. Improvement of efficacy and safety profile of simvastatin in comparison to reference product (zocor tablets) using nanoparticulate formulation approach. Int J Curr Pharm Res 2016;8:39-47.

24. Miyama M, Yang Y, Yasuda T, Okuno T, Yasuda H. Static and dynamic contact angles of water on polymeric surfaces. Langmuir 1997;13:5494-503.

25. Issa $\mathrm{M}$, Ferraz $H$. Intrinsic dissolution as a tool for evaluating drug solubility in accordance with the biopharmaceutical classification system. Dissolution Technol 2011;6-13. DOI:10.14227/DT180311P6

26. Palla BJ, Shah DO. Stabilization of high ionic strength slurries using the synergistic effects of a mixed surfactant system. J Colloid Interface Sci 2000;223:102-11.

27. Liu T, Hao J, Yang B, Hu B, Cui Z, Li S. Contact angle measurements: an alternative approach towards understanding the mechanism of increased drug dissolution from ethylcellulose tablets containing surfactant and exploring the relationship between their contact angles and dissolution behaviors. AAPS PharmSciTech 2018;19:1582-91.

28. Liu C, Liu Z, Chen Y, Chen Z, Chen H, Pui Y, et al. Oral bioavailability enhancement of $\beta$-lapachone, a poorly soluble fast crystallizer, by cocrystal, amorphous solid dispersion, and crystalline solid dispersion. Eur J Pharm Biopharm 2018;124:73-81. 